\title{
Corpus
}

6 | 2007

Interprétation, contextes, codage

Jacques Guilhaumou - Discours et événement. L'histoire langagière des concepts. Besançon : Presses Universitaires de Franche-Comté, 2006, 241 pages, $(12 €)$.

\section{Damon Mayaffre}

\section{OpenEdition}

Journals

Édition électronique

URL : http://journals.openedition.org/corpus/1422

DOI : $10.4000 /$ corpus. 1422

ISSN : $1765-3126$

Éditeur

Bases; corpus et langage - UMR 6039

Édition imprimée

Date de publication : 1 décembre 2007

Pagination : 193-198

ISSN : 1638-9808

Référence électronique

Damon Mayaffre, " Jacques Guilhaumou - Discours et événement. L'histoire langagière des concepts. Besançon : Presses Universitaires de Franche-Comté, 2006, 241 pages, (12 €). », Corpus [En ligne], 6 | 2007, mis en ligne le 02 juillet 2008, consulté le 24 septembre 2020. URL : http:// journals.openedition.org/corpus/1422 ; DOI : https://doi.org/10.4000/corpus.1422

Ce document a été généré automatiquement le 24 septembre 2020.

(c) Tous droits réservés 


\title{
Jacques Guilhaumou - Discours et événement. L'histoire langagière des concepts. Besançon : Presses Universitaires de Franche-Comté, 2006, 241 pages, $(12 €)$.
}

\author{
Damon Mayaffre
}

1 Après Discours et Archive. Expérimentations en analyse de discours co-signé en 1994 avec Denise Maldidier et Régine Robin, le dernier ouvrage de Jacques Guilhaumou, Discours et événement. L'histoire langagière des concepts, est appelé à faire date dans la communauté d'analyse de discours.

D'un abord difficile, l'ouvrage, le long de 5 chapitres et d'une importante postface, se caractérise par trois objectifs ambitieux imbriqués.

3 L'objectif premier, affiché par l'auteur, est de prolonger l'analyse de discours traditionnelle vers des terres guère explorées dans l'hexagone d'une histoire langagière des concepts et d'une histoire des événements linguistiques, inspirées d'une part par l'école française mais plus encore par l'école anglophone et allemande. L'objectif second et sous-jacent de l'ouvrage est de retracer l'histoire de l'AD, dont Jacques Guilhaumou est acteur depuis plus de 30 ans, comme si l'histoire de cette discipline interprétative était, comme l'a montré [Mazière 2005] partie intégrante de sa définition. Enfin, au terme de ce déplacement conceptuel de l'objectif de l'AD et du parcours historiographique sur la discipline, une ambition plus importante perce partout pour s'affirmer dans la conclusion: mener une réflexion philosophique avec Habermas, Koselleck, Foucault, Eco, Ricoeur, Deleuze et quelques autres sur le rapport entre les mots et les choses, le langage et la réalité, l'abstrait et le sensible, enfin et surtout sur l'événement, de chair et de langue, en tant qu'objet majeur d'une science humaine globale. 
4 Le chapitre introductif (pp. 11-43) est à la fois classique et précieux. Il retrace en trois temps et trois décennies les rapports entre histoire et linguistique noués au sein de l'analyse de discours en France depuis les années 1970. Fidèle à son statut d'historien linguiste, Jacques Guilhaumou montre comment, en France, le discours a pu d'abord être qualifié « d'objet d'histoire ", puis servir de pont interdisciplinaire entre linguistes et historiens dans une perspective interprétative, avant d'être enfin considéré en luimême pour lui-même (si l'expression est autorisée en parlant du discours) à condition de ne jamais le déraciner d'une réalité historique dont il est à la fois constituant et constitutif. Au final, c'est bien une « analyse de discours du côté de l'histoire, au titre de la prédominance de l'approche configurationnelle » (p.19) qui est retracée en soulignant son évolution.

5 Naïve dans son approche, au début les années 1970, durant lesquelles il convenait avant tout d'étudier la co-variance entre la réalité historique d'une part et le discours d'autre part, c'est-à-dire entre modèles sociaux d'un côté et structure linguistique de l'autre (p.13), l'analyse de discous a en effet vite évolué. Guilhaumou note par exemple que très rapidement une "relative autonomie des effets discursifs face aux réalités sociales » a été reconnue (p. 17). Plus loin, dans les années 1980, le fait discursif devient lui-même événement (p.19), cessant d'être le réceptacle d'une histoire qu'il rapporterait, pour devenir son acteur sinon unique en tout cas principal. Par là, c'est le statut de l'archive même qui se trouve modifié. L'archive est abordée désormais « dans le mouvement même de sa formation » (p. 21) car elle ne se contente pas de "figurer » fidèlement une réalité objective mais la "con-figure » en proposant un certain ordre des choses, des trajets thématiques orientés et en se montrant partie prenante de la réalité dont elle rend compte. En un mot définitif: «Il en ressort que le contexte interprétatif d'un énoncé [au sein de l'archive] n'est pas extérieur à sa description » (p. 21).

6 Une nuance forte, ici, doit néanmoins être apportée : en affirmant progressivement, mais définitivement à partir des années 1990, que les ressources interprétatives des textes se situent en leur sein, Jacques Guilhaumou se revendique sans réserve du «tournant interprétatif» mais garde une certaine réserve vis-à-vis du «linguistic turn » sur la base de la nécessaire connexion empirique entre la réalité et le discours (voir la Postface). Sa posture d'historien lui interdit en effet, face au texte, de franchir le rubicon qui l'empêcherait de retourner à un réel structuré certes par le langage, mais aussi en partie extérieur à lui. Si Guilhaumou se propose de montrer l'intrication entre le monde et ce langage qui est la condition de son interprétation, c'est aussi pour refuser « toute confusion entre la réalité sociale et les faits discursifs » (p. 41).

7 Le chapitre I (pp. 43-86) aborde de front l'histoire des concepts que la tradition française en analyse de discours a trop longtemps ignorée. De fait, Jacques Guilhaumou quitte la bibliographie hexagonale pour nous déplacer vers la bibliographie anglophone et plus encore germanique; et si l'ouvrage ne devait avoir qu'un seul intérêt pratique, la vulgarisation des apports anglo-saxons en $\mathrm{AD}$ serait cet intérêt-là.

8 A distance d'une histoire des représentations qui n'accorde guère d'importance à l'intentionnalité historique, donc à la manière dont l'acteur est partie intégrante du jugement et de la critique d'une société, l'histoire conceptuelle s'applique à étudier le rôle même du langage (lui-même situé histori-quement) dans la formation des concepts (socio-politiques par exemple). L'histoire des concepts en effet «s'interroge sur la 
connexion empirique entre la réalité et le discours, donc sur le rôle du contexte dans ses modes de compréhension et d'explication des phénomènes conceptuels. » (p. 44).

Deux courants participent à cette réflexion. Le principal est germanique, dont l'auteur majeur, désormais disponible en français, est Reinhart Koselleck. Pour ce dernier, selon Guilhaumou, l'objectif est de "prendre en compte le langage en acte dans tout processus de compréhension historique.»(p.47). Ou: "En résumé, la dimension historique $\mathrm{du}$ fait social relève en grande part de ses conditions langagières d'apparition sans s'y confondre» (p.48). Le second courant est anglophone avec "l'école de Cambridge». Ses figures de proue sont John G. A. Pocock et Quentin Skinner. Même si les deux auteurs diffèrent sur certains points, ils insistent l'un et l'autre sur la performativité du langage dans l'histoire telle qu'elle se déroule et telle qu'elle s'écrit. L'auteur (le contemporain dans un premier temps comme l'historien ensuite) est fondamentalement un acteur d'une histoire qu'il contribue à construire en nommant.

Ici, nous noterons que si Guilhaumou regrette globalement le retard français en matière d'histoire conceptuelle et la tendance au repli hexagonal de l'AD, il relève aussi un projet mis en chantier précocement par Saint Cloud qui peut servir de pont entre l'école française et l'école anglo-saxonne: les huit volumes du grand Dictionnaire des usages socio-politiques du français contemporain.

11 Après l'histoire conceptuelle, Jacques Guilhaumou prolonge la réflexion dans le chapitres II (pp. 87-120) et le chapitre III (pp. 121-154) en abordant les notions complexes et centrales de l'ouvrage: l'événement linguistique, l'événement discursif puis le récit d'événement. L'approche se fait de manière théorique mais aussi pratique par un réel travail sur l'archive: la langue du $18^{\text {ème }}$ révolutionnaire, le cas de "Tiers-Etat", d' "Assemblée Nationale » ou encore de la narration de la mort de Marat viennent illustrer concrètement la pensée théorique d'ensemble.

12 On rendra ici compte de la réflexion d'envergure préalable au développement, laissant le lecteur prolonger sa lecture pour le détail de la démonstration. Guilhaumou pose d'abord, dans une perspective phénoménaliste, que « les données empiriques n'ont pas de contenu préexistant à leur émergence perceptive » (p.89), et, pour l'auteur, le langage, précisément, est consubstantiel de cette émergence perceptive. Puis, s'appuyant sur une citation de Benoist, Guilhaumou pousse plus loin: «Si les choses sont 'données', il n'y a pas à proprement parler de choses dans le monde, mais rien que des 'événements' ( (p. 90), introduisant ainsi le statut cognitif de l'événement. Enfin, Guilhaumou peut préciser le projet : « de l'événementialité, il est donc question ici sur le mode de la donation linguistique : ce qui est donné ne peut être séparé de ce qui est dit, ce qui est dit a une existence pour nous, est donné par le seul fait d'être dit.» (p. 90).

13 Dès lors, la conclusion programmatique peut s'énoncer en ces termes avant d'être développée le long des deux chapitres: "Considérant premièrement que tout donné procède d'un événementialité, en second lieu que la donation linguistique est première du point de vue empirique adopté, nous pouvons nous intéresser au déploiement de la pluralité initiale de l'être en des points singuliers qualifiés d'événements linguistiques». (p. 92).

Le chapitre IV, L'archéologie de l'idée de progrès (XVI'-XVIII ${ }^{\circ}$ siècles) (pp. 155-178) est à la fois en continuité, et en décalage du propos principal, dans la mesure où il présente une 
réflexion sur la temporalité et les temporalités, en préalable d'une réflexion en cours sur la rupture des temps modernes dans la perspective de l'histoire conceptuelle.

Enfin, la Postface. La connexion empirique entre la réalité et le discours (pp. 189-221) propose de verser les pensées développées dans l'ouvrage au débat qui secoue la communauté linguistique depuis l'exhumation des manuscrits inédits de Saussure en 2002. Ce « retour à Saussure » (p. 189) apparaît à la fois fécond et justifié par l'utilisation même que Saussure fait du syntagme "événements linguistiques» (p. 193) et par l'idée sousjacente chez le Genevois d'une langue empirique, de caractère social (p.191) - donc historique - qui, pas moins que la structure, est l'objet du linguiste historien.

Si le lecteur dépasse l'écriture ardue de Jacques Guilhaumou, il tirera bénéfice de Discours et événement pour au moins trois raisons. D'abord, l'ouvrage est actuellement, en France, un des seuls qui croisent de manière documentée l'approche française et l'approche anglo-saxonne. Et cette ouverture géographique, absente dans [Mazière 2005], marginale dans [Charaudeau et Maingueneau 2002], se double d'une vision historique large qui situe l'actualité de l'AD dans un temps désormais long de plusieurs décennies. Ensuite, dans le cadre d'une AD souvent présentée comme un carrefour disciplinaire, l'ouvrage est un des rares, selon le distinguo de [Darbellay 2005], à être non seulement interdisciplinaire mais transdisciplinaire : la fécondation de l'histoire par la linguistique - et vice versa - aboutit à une pensée originale au-delà même des disciplines et nous rappelle que le discours, les concepts, l'événement, comme finalement tout objet de connaissance un peu complexe, n'appartiennent pas à des chapelles et ne peuvent être appréhendés que dans une approche globale dans laquelle les disciplines traditionnelles se subliment. Enfin et surtout, là où l'Analyse de Discours n'est souvent qu'une pratique ou une boite à outils pour traiter des textes, l'ouvrage de Jacques Guilhaumou tente de donner une assise épistémologique et un débouché philosophique à une discipline définitivement, selon l'auteur, à vocation herméneutique.

\section{BIBLIOGRAPHIE}

Charaudeau P. \& Maingueneau D. (2002). Dictionnaire d'analyse de discours. Paris : Seuil.

Darbellay F. (2005). Interdisciplinarité et transdisciplinarité en analyse des discours. Complexité des textes, intertextualité et transtextualité. Genève : Slatkine.

Guilhaumou J., Maldidier D. \& Robin R. (1994). Discours et Archive. Expérimentations en analyse de discours. Liège : Mardaga.

Mazière F. (2005). L'analyse de discours. Paris : PUF.

Saussure F. de (2002). Ecrits de linguistique générale. Paris : Gallimard. 